\title{
Impact of the Tobacco Treatment Guidelines for High Risk Groups (TOB.g): A pilot study among physicians specializing in CVD, Diabetes and COPD
}

\author{
Antigona C. Trofor', Sophia Papadakis, ${ }^{2,3}$, Constantine I. Vardavas, $4,5,6$, Lucia Maria Lotrean7, Cristina-Maria \\ Gavrilescu', Vaso Evangelopoulou ${ }^{5,6}$, Theodosia Peleki ${ }^{5}$, Letitia Trofor ${ }^{1 *}$, Panagiotis K. Behrakis ${ }^{4,6,6,8}$
}

\begin{abstract}
INTRODUction The 2017 Tobacco Cessation Guidelines for High-risk Groups (TOB-G) is a comprehensive document on best practices for smoking cessation in clinical practice. The purpose of this pilot study was to assess physician satisfaction and changes in tobacco-related knowledge, selfefficacy and attitudes following exposure to training in the TOB-G guidelines for patients with cardiovascular disease (CVD), chronic obstructive pulmonary disease (COPD), or diabetes.

METHODS A pre-post pilot study was conducted. All participating physicians received an electronic or printed copy of the TOB-G guidelines for patients with GVD, COPD or diabetes. Physicians were also exposed to a one-day training focused on the key clinical practice recommendations from the TOB.g guidelines. Outcome measurement occurred via survey before, immediately following and 6 months after exposure to the training.

RESULTS Fifty physicians participated in the TOB.g training session. High rates of participant satisfaction were documented (exceeded expectations $47.7 \%$; met expectations to a great extent $52.3 \%$ ). Significant increases in physician knowledge and self-efficacy were documented immediately following and 6 months after exposure to the guideline training session. Exposure to the training was associated with positive changes in some but not all tobacco-related treatment attitudes, however these were no longer significant at the 6-month follow-up. Lower knowledge, confidence and unfavourable attitudes were documented for aspects of treatment related to pharmacotherapy.

CONCLUSIONS Positive changes in previously reported barriers to the delivery of tobacco treatment among physicians were documented following exposure to the TOB.g guidelines and training for patients with CVD, COPD or diabetes.
\end{abstract}

\section{AFFILIATION}

1 University of Medicine and Pharmacy Grigore T. Popa, lasi, Romania

2 Division of Prevention and

Rehabilitation, University of Ottawa

Heart Institute, Ottawa, Canada

3 Faculty of Medicine, University of

Ottawa, Ottawa, Canada

4 Institute of Public Health, American

College of Greece, Athens, Greece

5 George D. Behrakis Research Lab,

Hellenic Cancer Society, Athens, Greece

6 Hellenic Centre for Disease Control and

Prevention, Athens, Greece

7 Iuliu Hatieganu University of Medicine and Pharmacy, Cluj-Napoca, Romania

8 Biomedical Research Foundation of the Academy of Athens (BRFAA), Athens,

Greece

*PhD student

CORRESPONDENCE TO

Lucia Lotrean. Iuliu Hatieganu Universityof Medicine and Pharmacy,

Cluj-Napoca,Romania. E-mail: Ilotrean@ gmail.com

\section{KEYWORDS}

tobacco treatment, Europe, guidelines, cardiovascular, diabetes, Chronic Obstructive Pulmonary Disease

Received: 20 December 2017

Revised: 20 March 2018

Accepted: 20 March 2018

\section{INTRODUCTION}

Patients with cardiovascular disease (CVD), diabetes (DM) and chronic obstructive pulmonary disease (COPD) who smoke constitute a high-risk population of patients. There is strong evidence to show that tobacco use exponentially increases morbidity, mortality and quality of life in these patient populations ${ }^{1,2}$.

Smoking cessation is recognized as the single most powerful intervention for the secondary prevention 
of GVD, COPD, and this could be argued for patients with Type II Diabetes ${ }^{2-4}$. Smoking cessation produces a significant reduction in the progression of existing disease, reduces hospital re-admissions, improves response to therapy, and results in lower morbidity and all-cause mortality ${ }^{2,3,5-7}$. Given this, smoking cessation should be a key priority for the secondary prevention of these chronic diseases and should be treated with the same rigour as other major risk factors ${ }^{1}$. Providers have identified a lack of knowledge/confidence as a major barrier to the delivery of tobacco dependence treatment to patients $^{8-10}$.

The Tobacco Cessation Guidelines for High-risk Groups (TOB.g) were developed by a scientific panel of experts in order to inform clinical practice of tobacco dependence treatment among five high-risk patient populations including: adolescents, pregnant women, patients with cardiovascular disease (CVD), chronic obstructive pulmonary disease (COPD), and diabetes ${ }^{1,12}$. The guidelines review pathophysiology and evidence related to tobacco's effects on disease course and treatment strategies for each of the high-risk patient populations and presents clinical practice recommendations. The GRADE evidence grading system has been used to rate the quality of evidence supporting each of the clinical practice recommendations presented in the TOB.g guidelines ${ }^{13}$. The TOB.g guidelines are available online at http://tob-g.eu/. The TOB.g guidelines recommend that all health care professionals including cardiologists, respirologists, endocrinologists, internists, general practitioners, psychiatrists, and nurses, should be trained in the latest evidence-based tobacco treatments and be prepared to intervene with their patients who smoke $^{13}$.

The purpose of this pilot study was to assess physician satisfaction and changes in tobacco-related knowledge, self-efficacy, and attitudes, following exposure to training in the TOB-G guidelines for patients with cardiovascular disease (CVD), chronic obstructive pulmonary disease (COPD) or diabetes.

\section{METHODS}

\section{Design}

A pre-post pilot study was conducted among a sample of physicians. Measurement occurred before (Time
1), immediately following (Time 2 ) and 6 months after (Time 3 ) exposure to the TOB.g guidelines and to a 1-day training intervention. The present evaluation focused specifically on the clinical practice recommendations for patients with CVD, COPD or diabetes. Separate evaluations were conducted for the adolescent and obstetrics populations that were reported elsewhere.

\section{Setting and study population}

This pilot intervention took place in Iasi, Romania. Physicians involved in the treatment of patients with CVD, COPD or diabetes were invited to attend the TOB.g training session. In order to participate in the study, individuals were required to meet the following eligibility criteria: 1) be a licenced physician, 2) work directly with patients with either GVD, COPD or diabetes, 3) be willing to participate in the TOB.g guideline training session. Ethics approval for the pilot TOB.g was received from the Ethics Board of the Pulmonary Diseases Hospital in Iasi, Romania.

\section{Intervention}

A 1-day training session was conducted in Iasi, Romania on 11 October 2016. The training session was designed to communicate key recommendations from the TOBG guidelines and the specific clinical practice recommendations for patients with GVD, COPD or diabetes. All participants received a hard copy of the TOB-G guideline summary at the training session and an electronic copy after the session ${ }^{1}$. The training curriculum included an overview of the pathophysiology of tobacco use among patients with CVD, COPD or diabetes, presenting information on: prevalence of tobacco use in these populations, health risk of tobacco use, health benefits of cessation, challenges to cessation among these high risk populations of patients, overview of evidencebased counselling and pharmacological treatment strategies, and how the TOB.g clinical practice recommendations strategies can be implemented into clinical practice in both the hospital and outpatient settings. Specifically, this included an overview of the recommended 5As (ask, advise, assess, assist, arrange) model for supporting smoking cessation in a clinical setting, counselling techniques for delivering effective advice to quit tailored to the specific 
patient populations, motivational interviewing techniques, counselling for individuals ready to quit, recommendations for pharmacotherapy, and follow-up in these patient populations. Case studies and role-play was used as part of the training. The training program was delivered by an international team of clinical experts and tobacco treatment specialist involved in co-authorship of the TOB-G guideline chapters.

\section{Procedures}

Specialists in pulmonary diseases, cardiology, internal medicine, psychiatry and general practitioners were invited to participate in the TOB.g training. All eligible participants provided a written informed consent. Consenting participants completed a provider survey immediately before (Time 1) the TOB.g training session that assessed: provider demographic characteristics, tobacco treatment knowledge, tobacco treatment selfefficacy, and current rates of tobacco treatment delivery. At the end of the training day (Time 2), participants completed a post-training survey to measure the immediate impact of the training session on outcomes of interest. Participants also completed the 6-month follow-up survey (Time 3) following the completion of the training program. The 6-month survey was sent by email or by post to participants. Two email reminders were sent, on the 7th and 14th day following the surveys' distribution, to anyone who had not responded and a phone call was made to anyone who had not responded to these reminders, before categorizing them as lost to follow-up.

A total of 60 physicians were invited to participate in the TOB.g pilot study and 50 participated in the training session (participation rate was $83.3 \%$ ). The primary reasons for non-participation were a lack of interest $(n=8)$ and annual leave $(n=2)$. The followup rate was $94.4 \%$ and $92.0 \%$, respectively, at posttraining assessment (Time 2) and 6-month follow-up (Time 3 ). The recruitment flow diagram is presented in Figure 1.

\section{Measures}

Physician's knowledge was assessed using a 20 -item knowledge assessment questionnaire. All knowledge questions were developed from information
Figure 1. Recruitment flow of the health care practitioners in the TOB-G, CVD, COPD and Diabetes Pilot, Iasi, Romania 2016

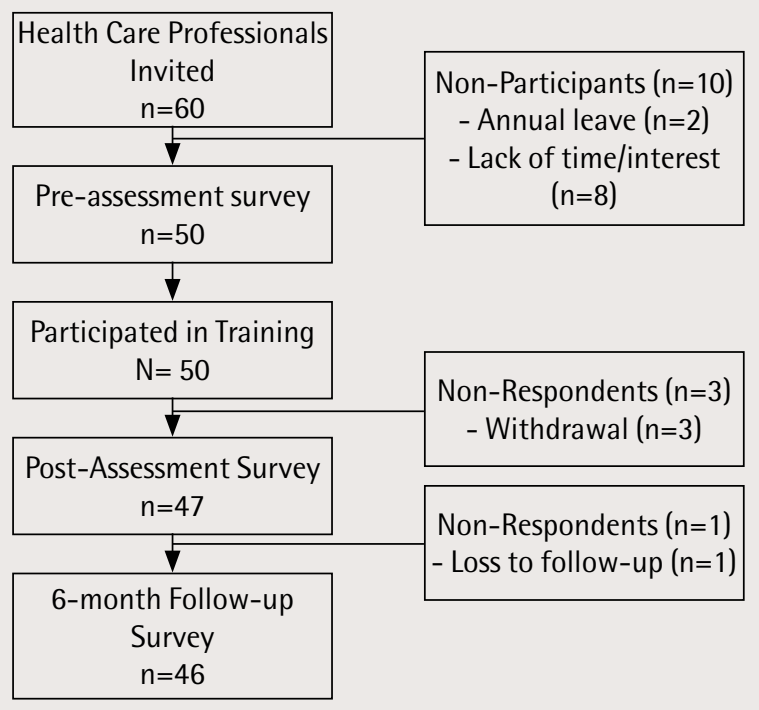

contained in the 2017 TOB.g Tobacco Treatment Guidelines and presented as part of the training intervention ${ }^{1}$. The questions included general information on tobacco treatment and knowledge questions related to each of three high risk patient populations (CVD, COPD, Diabetes). A 6-item questionnaire was used to assess participant's selfefficacy (i.e. confidence) in the delivered tobacco treatment interventions using the question ' $O n$ a scale of 1 to 10, how would you describe your confidence in the following areas ( 1 - being not at all confident to 10 - being very confident)'. Selfefficacy has been shown to be highly correlated to rates of tobacco treatment delivery ${ }^{14,15}$. Participants' attitudes (10-items) related to recommendations and content communicated in the guideline training were assessed (i.e. role of health care professionals in tobacco treatment, recommendations regarding the integration of $5 \mathrm{As}$ into clinical routines, the efficacy and safety of pharmacotherapies). Responses were recorded using a 5-point Likert's scale with 1 -strongly disagree to 5 -strongly agree. The survey questions for self-efficacy and attitudes were based on an existing tool ${ }^{16,17}$ and built on the work of others ${ }^{18,19}$. Participants' satisfaction with the guideline training was assessed at the post-training assessment (Time 2) using only three questions: 'Overall to what extent did this workshop meet your 
expectations'; with response options being: 'did not meet my expectations', 'to some extent', 'to a great extent', 'exceeded my expectations'. Additionally, participants were also asked to identify what aspects of the guidelines, training program and areas that required improvement, using free text.

\section{Statistical analysis}

Descriptive statistics were used to summarize participant characteristics. In order to evaluate the effectiveness of the TOBG guidelines on a participant's knowledge, responses were converted to dichotomous data $(1=$ correct response, $0=$ incorrect response). Chi-squared analysis was performed to compare $\mathrm{T} 1$ vs $\mathrm{T} 2$, and $\mathrm{T} 1$ vs $\mathrm{T} 3$, for each knowledge question. For the analysis of continuous data for self-efficacy and intentions, paired sample t-tests were performed. Statistical significance was set at 0.05. Missing data were not replaced. Statistical analysis was performed with the use of Statistical Package for Social Sciences (SPSS) 24th edition (Canada). We examined using univariate analysis, and as appropriate regression analysis, the possible association between demographic characteristics of the sample and outcomes of interest. Qualitative data regarding measures of provider satisfaction were assessed using thematic analysis with information coded by two members of the research team and key themes extrapolated.

\section{RESULTS}

\section{Participant's characteristics}

Table 1 depicts the sociodemographic characteristics of participants. The majority of participants were female $(84.0 \%)$, under 50 years of age $(80.0 \%)$ and worked in a hospital $(80.0 \%)$ in an urban setting $(90.0 \%)$. The professional training of the majority of participants was respirology $(60.0 \%)$. Participants had an average of $11.0( \pm 9.64)$ years of clinical practice experience and reported seeing an average of $46.4( \pm 41.16)$ patients per week in their clinical practice. The majority of participants (78.0\%) had not previously participated in smoking cessation training. Among the physicians sampled, $66.0 \%$ had never smoked and $22.0 \%$ reported current tobacco use. The majority $(62.5 \%)$ of participants identified that over of $40 \%$ of their patients were smokers.
Table 1. Descriptive characteristics of participants $(\mathrm{n}=50)$, Iasi Romania 2016

\begin{tabular}{|c|c|c|}
\hline Variable & n & $\%$ \\
\hline \multicolumn{3}{|l|}{ Gender } \\
\hline Male & 8 & 16.0 \\
\hline Female & 42 & 84.0 \\
\hline \multicolumn{3}{|l|}{ Age (Years) } \\
\hline$<29$ & 8 & 16.0 \\
\hline $30-39$ & 17 & 34.0 \\
\hline $40-49$ & 15 & 30.0 \\
\hline $50-59$ & 9 & 18.0 \\
\hline $60-69$ & 1 & 2.0 \\
\hline \multicolumn{3}{|l|}{ Professional training } \\
\hline Respirologist & 30 & 60.0 \\
\hline Cardiologist & 2 & 4.0 \\
\hline Internal Medicine & 5 & 10.0 \\
\hline General Medicine & 6 & 12.0 \\
\hline Geriatrics & 2 & 4.0 \\
\hline Psychiatry & 5 & 10.0 \\
\hline \multicolumn{3}{|l|}{ Practice setting } \\
\hline Solo or group practice & 6 & 12.0 \\
\hline Hospital & 40 & 80.0 \\
\hline Primary Care & 2 & 4.0 \\
\hline Ambulatory & 2 & 4.0 \\
\hline \multicolumn{3}{|l|}{ Area of practice } \\
\hline Urban & 45 & 90.0 \\
\hline Rural & 5 & 10.0 \\
\hline $\begin{array}{l}\text { Average patients per week, } \\
\text { mean (SD) }\end{array}$ & $45.8(40.9)$ & \\
\hline Years of practice, mean (SD) & $11.3(9.9)$ & \\
\hline \multicolumn{3}{|l|}{ Employment status } \\
\hline Part-time & 3 & 6.0 \\
\hline Full-time & 47 & 94.0 \\
\hline \multicolumn{3}{|c|}{ Previous participation in a smoking cessation program } \\
\hline Yes & 11 & 22.0 \\
\hline No & 39 & 78.0 \\
\hline \multicolumn{3}{|c|}{ Personal experience with the tobacco use } \\
\hline Current smoker & 11 & 22.0 \\
\hline Former smoker & 6 & 12.0 \\
\hline Non smoker & 33 & 66.0 \\
\hline
\end{tabular}

\section{Provider knowledge}

Table 2 presents a summary of the percentage of participants who responded correctly to each of the knowledge questions assessed. At baseline, participants demonstrated high rates of knowledge for most of the areas assessed. However, low rates of knowledge were noted, at baseline, for questions related the use of pharmacotherapy, typical duration of cravings, the need for glucose monitoring among diabetic patients who quit smoking, risk reduction 
Table 2. Percentage (\%) of physicians who responded correctly to knowledge assessment before and after exposure to the TOB.g guidelines and training in Iasi, Romania, 2016

\begin{tabular}{|c|c|c|c|c|c|}
\hline \multirow[b]{2}{*}{ Knowledge Question } & \multicolumn{3}{|c|}{$\begin{array}{l}\text { Per cent }(\%) \text { correct } \\
\text { response }\end{array}$} & \multicolumn{2}{|c|}{ p-value } \\
\hline & $\begin{array}{l}\text { Pre } \\
(T 1)\end{array}$ & $\begin{array}{l}\text { Post } \\
(\mathrm{T} 2)\end{array}$ & $\begin{array}{l}\text { 6-month } \\
\text { FU (T3) }\end{array}$ & $\begin{array}{c}\text { TI vs } \\
\text { T2 }\end{array}$ & $\begin{array}{l}\text { T2 vs } \\
\text { T3 }\end{array}$ \\
\hline 1. Is it safe to continue to smoke while using NRTs? & 20.5 & 73.3 & 87.0 & 0.031 & 0.000 \\
\hline 2. Which are the most effective medications in terms of increase success rates? & 4.10 & 37.0 & 34.8 & 0.000 & 0.000 \\
\hline 3. How long does a craving typically last? & 20.5 & 87.0 & 76.1 & 0.000 & 0.000 \\
\hline 4. What is the most common side effect of varenicline? & 34.0 & 82.6 & 71.7 & 0.000 & 0.000 \\
\hline 5. Nicotine replacement therapies are contraindicated for people with CVD & 67.3 & 100.0 & 91.3 & 0.000 & 0.027 \\
\hline 6. Nicotine is as addictive as other drugs such as heroin or cocaine & 85.4 & 97.8 & 93.5 & 0.125 & 0.210 \\
\hline $\begin{array}{l}\text { 7. The most effective treatment for reducing the rate of COPD progression among patients } \\
\text { who smoke is... }\end{array}$ & 74.5 & 80.4 & 91.3 & 0.453 & 0.018 \\
\hline 8. Respiratory symptoms ameliorate after ... after quitting smoking & 32.7 & 63.0 & 58.7 & 0.017 & 0.013 \\
\hline $\begin{array}{l}\text { 9. Which of the following therapies is the most effective for smoking cessation in COPD } \\
\text { smokers? }\end{array}$ & 71.4 & 65.2 & 91.3 & 0.664 & 0.006 \\
\hline 10. Smokers with COPD find it more difficult to stop using tobacco, due to, except one: & 65.3 & 65.2 & 80.4 & 1.000 & 0.109 \\
\hline $\begin{array}{l}\text { 11. Which of the following secondary prevention measures results in the greatest reduction } \\
\text { of mortality in patients with coronary heart disease: }\end{array}$ & 70.8 & 82.6 & 91.3 & 0.289 & 0.010 \\
\hline $\begin{array}{l}\text { 12. The increase in the risk of morbidity and mortality caused by coronary heart disease for } \\
\text { persons exposed to second hand smoking is: }\end{array}$ & 57.1 & 82.2 & 80.4 & 0.021 & 0.002 \\
\hline $\begin{array}{l}\text { 13. Smoking cessation provided by primary care physicians for patients with cardiovascular } \\
\text { disease is... }\end{array}$ & 93.9 & 97.8 & 100.0 & 0.625 & 0.083 \\
\hline 14. Smoking cessation after myocardial infarction reduces cardiovascular mortality by: & 30.6 & 54.3 & 84.8 & 0.021 & 0.000 \\
\hline $\begin{array}{l}\text { 15. A behavioral intervention in patients hospitalized with cardiovascular disease is } \\
\text { indicated: }\end{array}$ & 98.0 & 100.0 & 100.0 & 1.000 & 0.323 \\
\hline 16. The excess risk of cardiovascular disease in elderly smokers is & 92.0 & 100.0 & 100.0 & 0.125 & 0.083 \\
\hline 17. Smoking among diabetic patients amplifies the risk of... & 54.0 & 91.3 & 66.7 & $<0.001$ & 0.229 \\
\hline $\begin{array}{l}\text { 18. Due to possible deterioration in glycemic control in the first ......... after quitting, } \\
\text { clinicians should closely monitor glycaemia and adjust anti-diabetic medications to } \\
\text { maintain effective glycemic control following smoking cessation. }\end{array}$ & 8.2 & 58.7 & 63.0 & $<0.001$ & 0.000 \\
\hline $\begin{array}{l}\text { 19. Which of the following therapies is the most efficient for smoking cessation in Diabetic } \\
\text { smokers: }\end{array}$ & 93.6 & 91.3 & 95.7 & 1.000 & 0.660 \\
\hline $\begin{array}{l}\text { 20. Due to the increased risk of ...... bupropion is not recommended for use among DM } \\
\text { patients using hypo-glycemic agents or insulin }\end{array}$ & 36.2 & 54.8 & 65.2 & 0.012 & 0.006 \\
\hline
\end{tabular}

Proportions represent the $\%$ of providers who responded correctly to the knowledge question at each time point. p-values were calculated using chi-squared statistics *Only those practitioners with data at both the pre- and post assessment were included in analysis.

for CVD patients and respiratory symptom reduction following smoking cessation. Significant increases in knowledge were documented for 19/27 knowledge questions; these differences were statistically significant for 14 knowledge questions. For questions for which baseline knowledge was poor, very large jumps in the proportion of respondents who answered correctly were observed at postassessment. In the case of four of the knowledge questions, respondents were correct more than $90 \%$ of the time at baseline and no further significant increases were documented.

\section{Self-efficacy}

There was a significant increase in confidence among participants following the TOB.g training in all six areas assessed immediately following the training and changes observed remained significant at the 6-month follow-up (Table 3). At baseline, the lowest rates of self-efficacy across the six domains assessed was documented for confidence in prescribing a quit smoking medication. While a significant increase in this self-efficacy domain was seen following exposure to the TOB.g training, confidence in prescribing a quit smoking 
Table 3. Participants self-efficacy and attitudes related to evidence-based tobacco treatment delivery before and after the TOB.g training program, Iasi Romania 2016

\begin{tabular}{|c|c|c|c|c|c|}
\hline \multirow[b]{2}{*}{ Variable } & \multicolumn{3}{|c|}{ Vean Score } & \multicolumn{2}{|c|}{ p-value } \\
\hline & $\begin{array}{l}\text { Pre } \\
(T 1)\end{array}$ & $\begin{array}{l}\text { Post } \\
\text { (T2) }\end{array}$ & $\begin{array}{l}\text { 6- month } \\
\text { FU (T3) }\end{array}$ & TI us T2 & TI vs T3 \\
\hline \multicolumn{6}{|l|}{ Self-Efficacy ${ }^{a}$} \\
\hline Advising patients to quit smoking & 8.19 & 9.49 & 8.87 & 0.000 & 0.002 \\
\hline Providing brief smoking counseling ( $<3$ minutes) & 7.42 & 9.17 & 8.48 & 0.002 & 0.002 \\
\hline Providing counseling to patients not motivated to quit & 6.65 & 8.73 & 8.61 & 0.000 & 0.000 \\
\hline Prescribing quit smoking medication & 4.23 & 6.16 & 6.20 & 0.000 & 0.002 \\
\hline Providing smoking cessation counselling ready to quit & 6.76 & 8.80 & 7.76 & 0.000 & 0.000 \\
\hline Arranging timely follow-up for patients planning to quit smoking & 5.77 & 8.83 & 7.83 & 0.000 & 0.000 \\
\hline \multicolumn{6}{|l|}{ Attitudes ${ }^{b}$} \\
\hline Helping my patients quit smoking is an important part of my role as a clinician (+) & 4.84 & 4.93 & 4.75 & 0.290 & 0.302 \\
\hline Clinicians should advise patients to quit smoking even if it's not the reason for the visit (+) & 4.80 & 4.89 & 4.89 & 0.323 & 0.323 \\
\hline Counselling by a clinician helps motivate smokers to quit (+) & 4.42 & 4.84 & 4.53 & 0.000 & 0.511 \\
\hline Clinicians should make appointments specifically to help patients quit (+) & 4.39 & 4.75 & 4.56 & 0.006 & 0.173 \\
\hline Smoking is a personal decision which does not concern the clinician (-) & 1.89 & 2.04 & 2.04 & 0.564 & 0.564 \\
\hline For many tobacco users smoking is an addiction (+) & 4.61 & 4.20 & 4.64 & 0.439 & 0.643 \\
\hline A patient's willpower alone is what will determine their success with quitting (-) & 3.26 & 3.21 & 3.37 & 0.844 & 0.331 \\
\hline First line smoking cessation pharmacotherapies work well in helping patients quit (+) & 3.86 & 4.16 & 4.02 & 0.000 & 0.578 \\
\hline $\begin{array}{l}\text { First line pharmacotherapies for smoking cessation are not safe and have side effects that } \\
\text { out weight their benefits (-) }\end{array}$ & 2.49 & 1.76 & 2.25 & 0.000 & 0.556 \\
\hline I have the required skills to help my patients quit smoking (+) & 3.30 & 4.23 & 3.91 & 0.000 & 0.003 \\
\hline
\end{tabular}

a Responses reported on a scale of 1 to 10; 1-not at all confident to 10-extremely confident. b Responses were recorded using a 5-point Likert's scale; 1-strongly disagree to 5-strongly agree. $\quad+=$ reflects a positive attitude and higher scores reflect desired direction of change. $\quad-=$ reflect a negative attitude and lower scores reflect the desired direction of change. p-values were calculated using paired t-tests. ${ }^{*}$ Only those practitioners with data at both the pre and post assessment were included in analysis.

medication remained lower than for other aspects of tobacco treatment delivery assessed (mean selfefficacy score: 4.23 at time 1, 6.16 at time $2 ; 6.20$ at time $3 ; \mathrm{p}<0.00)$. For all other aspects of tobacco treatment, the average self-efficacy score post training was greater than 8 out of 10 immediately following the training. At the 6-month assessment the average self-efficacy scores decreased slightly but remained significantly increased when compared to baseline.

\section{Provider attitudes}

Exposure to the training program was associated with significant increases in 5 of the 10 tobaccorelated attitudes assessed at the post-training assessment, however these were no longer significant at the 6-month follow-up (Table 3 ). A significant proportion of clinicians at baseline reported very positive attitudes and further statistically significant increases were not possible. A significant proportion of participants reported concerns about the safety of quit-smoking medications at baseline, and while there was a significant decrease post training in this negative attitude, no change was documented at the 6-month follow-up, suggesting a portion of participants had on-going concerns about the safety of these medications despite evidence regarding their safety contained in the TOB.g guidelines. There was also the belief, documented at baseline, that patient willpower alone would determine their success with quitting, which was not found to change post training. There were no significant differences documented for measures of knowledge, self-efficacy or attitudes based on participant's demographic characteristics. The only exception being the knowledge question regarding safety of nicotine replacement therapy (NRT) for patients with CVD, for which participants who smoke were more likely to respond incorrectly compared to non smokers at baseline $(\mathrm{p}=0.044)$.

\section{Satisfaction with TOB.g training}

Almost half of participants (47.7\%) stated that the 
TOB.g training received exceeded their expectations and $52.3 \%$ reported that the workshop met their expectations to a great extent. Participants identified training content related to motivational interviewing, counselling skills and the practical cases were most useful to their own practice. Ten per cent of participants indicated that they found it difficult to absorb all of the information presented in one day and suggested expanding the training to two days. Other recommendations received to improve the TOB.g training sessions were to include more sessions and presentations with role-play scenarios and examples and the inclusion of additional case studies that were specific to the high-risk patient groups. There were no significant differences in satisfaction with the training: between male and female participants, urban vs rural setting, professional background, or personal smoking status.

\section{DISCUSSION}

Clinical practice guidelines have an important role to play in influencing professional practice and in guiding the clinical treatment. This pilot study of a training program, based on the 2017 TOB.g guidelines, documented significant improvements in physician's tobacco treatment knowledge, selfefficacy and attitudes immediately following the training. Effects observed for physician self-efficacy immediately following the training intervention were maintained at the 6-month follow-up. Changes in physician attitudes were, however, not as distinct, which for the majority of attitudes assessed this can be explained by a ceiling effect due to very positive baseline response. For those attitudes for which significant effects were documented post training, differences observed were no longer significant at the 6-month follow-up, in particular those attitudes related to medication safety and patient willpower. There are several possible reasons for this, including the intervention did not adequately address these attitudes or that other sources of information are responsible for these attitudes including a clinician's own experiences.

Importantly, relative to other areas of tobacco treatment delivery, participants reported low confidence regarding prescribing evidence-based pharmacotherapy at baseline, and while confidence following the TOB-G training was significantly improved it remained lower than in other areas assessed. Our data examining physician attitudes suggest that concerns about the safety of these medications in high-risk populations also exist, and may be influencing participant's self-efficacy. Given that these medications have very strong randomized controlled trial (RCT) evidence supporting their effectiveness in increasing rates of tobacco treatment and that their safety has been established in high risk groups, it would be important for additional practice and research initiatives to examine methods for increasing physician confidence in the use of these medications in both the general population of tobacco users and in particular among patients with diabetes, COPD or cardiovascular disease ${ }^{20}$. Consideration should also be given to the amount of content covered in a 1-day training session and to alternate methods of delivering information to increase provider retention and satisfaction, as indicated in other studies $^{21}$.

While clinical practice guidelines, from international authorities for the management of CVD, COPD or diabetes, identify smoking cessation as an important clinical target, they do not necessarily provide sufficient depth to guide clinical practice in this high-risk group of patients ${ }^{22-24}$. The exception being the 2016 European Guidelines on Cardiovascular Disease Prevention in Clinical Practice, which offer more specific guidance on treating tobacco use in clinical practice ${ }^{25}$. The TOB.g guidelines, as such, is a complementary clinical practice guidance document for specialists working with patients with CVD, COPD or diabetes. Other groups have reported the value of provider tobacco treatment training in influencing practice behaviors, and have also found significant increases in outcomes of interest ${ }^{26-29}$. Specifically, a review by the Cochrane Collaboration on training health care professionals in smoking cessation found that training increased the likelihood that providers would perform tasks of smoking cessation than untrained controls, including: asking patients to set a quit date, make follow-up appointments, counselling of smokers, provision of self-help material and prescription of a quit date. There was a documented effect on patient point prevalence abstinence $(\mathrm{OR}=1.36,95 \% \mathrm{CI}: 1.20-1.55, \mathrm{p}=0.004)^{27} . \mathrm{A}$ 
systematic review by Mostofian reported that active forms of medical education were more effective for guideline implementation in clinical practice than passive approaches and when key opinion leaders are involved in the delivery of training ${ }^{26}$. These evidence-based training techniques were incorporated into the TOB.g training. Our study adds data to the important role of training health care professionals in clinical practice guidelines related to tobacco dependence treatment. It would be important to note that a review by Papadakis et al., which examined smoking cessation interventions in the primary care setting, reported training alone had a significant effect on both provider behaviors and patient cessation, and importantly that when training was combined with other patient and provider level interventions there was an increased effect size on these outcomes ${ }^{28}$. Low cost interventions, such as the development of guidelines and training, could be further enhanced if combined with additional supports such as electronic medical record prompts, adjunct counselling, and access to low cost quit smoking medications.

Our study is important for several reasons. First, patients with CVD, COPD or diabetes who smoke are priority populations for tobacco treatment ${ }^{1,30}$, despite this, low rates of intervention are reported in health care settings. Second, the majority of health care providers, including specialists working with highrisk populations, have not been trained in evidencebased tobacco treatment guidelines.

This study also has its limitations, as it was a pilot study involving one European country and a sample of fifty physicians, so replication of the study findings would be important. The lack of the control condition may also be considered a study limitation. The majority of participants were female and trained in respirology and as such may not be representative of the broader professional community. Sample size limitations meant detecting significant predictors of outcomes to be unlikely, and this may be an important question for future research. Our study used an existing tool to examine provider self-efficacy and knowledge, but it is possible that not all important aspects of these two intermediaries to clinical practice behaviors have been assessed. Additionally, all data collected were self-reported and subject to responder bias.

\section{CONCLUSIONS}

Our study found exposure to the TOB.g guidelines for High Risk Patients with GVD, COPD or diabetes was associated with increased physician knowledge of and confidence in the delivery of tobacco treatment in these priority populations. We know that both self-efficacy and attitudes are important predictors of future clinician behaviour ${ }^{15,16,18,19,31}$. The TOB.g guidelines and training represent two low-cost interventions, which have the potential to be widely disseminated.

\section{REFERENCES}

1. Behrakis P, Vardavas C, Papadakis S. (Eds.). Tobacco Cessation Guidelines for High Risk Groups. Athens: Hellenic Center for Disease Control and Prevention (KEELPNO); 2017.

2. USDHHS. The Health Consequences of Smoking-50 Years of Progress: A report of the Surgeon General. U.S. Department of Health and Human Services, 2015. http://www.surgeongeneral.gov/library/reports/50years-of-progress. Accessed December, 2017

3. Global Strategy for the Diagnosis, Management and Prevention of COPD. Global Initiative for Chronic Obstructive Lung Disease (GOLD), 2010. www. goldcopd.org Accessed December, 2017

4. Pan A, Wang Y, Talaei M, Hu FB. Relation of Smoking With Total Mortality and Cardiovascular Events Among Patients With Diabetes Mellitus A Meta-Analysis and Systematic Review. Circulation. 2015 Nov 10;132(19):1795-804. doi: 10.1161/CIRCULATIONAHA.115.017926

5. Tonstad S, J. AJ. Cardiovascular risks associated with smoking: a review for clinicians. Eur J Cardiovasc Prev Rehabil. 2006;13(4):507-14. doi: 10.1097/01.hjr.0000214609.06738.62

6. Mohiuddin SM1, Mooss AN, Hunter CB, Grollmes TL, Cloutier DA, Hilleman DE. Intensive smoking cessation intervention reduces mortality in high-risk smokers with cardiovascular disease. Chest. 2007;131(2):446-52. doi:10.1378/chest.06-1587

7. Critchley JA, Capewell S. Mortality risk reduction associated with smoking cessation in patients with coronary heart disease: a systematic review. JAMA. 2003;290(1):86-97. doi: 10.1001/jama.290.1.86

8. Vogt F, Hall S, Marteau TM. General practitioners' and family physicians' negative beliefs and attitudes towards discussing smoking cessation with patients: a systematic review. Addiction. 2005;100(10):1423-31. doi: 10.1111/j.1360-0443.2005.01221.x

9. Eldein HN, Mansour NM, Mohamed SF. Knowledge, Attitude and Practice of Family Physicians Regarding Smoking Cessation Counseling in Family 
Practice Centers, Suez Canal University, Egypt. J Family Med Prim Care. 2013;2(2):159-163. doi: 10.4103/2249-4863.117411

10. Jiménez-Ruiz CA, Andreas S, Lewis KE, et al. Statement on smoking cessation in COPD and other pulmonary diseases and in smokers with comorbidities who find it difficult to quit. Eur Respir J. 2015 ;46(1):61-79. doi: 10.1183/09031936.00092614

11. McIvor A, Kayser J, Assaad JM, Brosky G, Demarest P, Desmarais P, et al. Best practices for smoking cessation interventions in primary care. Can Respir J. 2009 JulAug;16(4):129-34. doi: 10.1155/2009/412385

12. Behrakis P. TOB-G: Tobacco Cessation Guidelines for High Risk Populations. Tob. Prev. Cessation 2016;2(April Supplement):39. doi: 10.18332/tpc/62428

13. Guyatt G, Oxman A, Vist GE, et al. GRADE: an emerging consensus on rating quality of evidence and strength of recommendations. BMJ. 2008;336(7650):924-926. doi: 10.1136/bmj.39489.470347.AD

14. O’Loughlin J, Makni H, Tremblay M, et al. 2001. Smoking cessation counseling practices of general practitioners in Montreal. Prev Med. 2001;33(6):627-38. doi:10.1006/pmed.2001.0937

15. Schnoll RA, Rukstalis M, Wileyto EP, and AE Shields. Smoking cessation treatment by primary care physicians: An update and call for training. Am J Prev Med 2006;31(3):233-239.

16. Papadakis S, Pipe AL, Reid RD, Tulloch H, Mullen K, Assi R, Cole A, Wells G. Effectiveness of performance coaching for enhancing rates of smoking cessation treatment delivery by primary care providers: Study protocol for a cluster randomized controlled trial. Contemp Clin Trials. 2015;45(Pt B):184-190. doi: 10.1016/j.cct.2015.08.013

17. Girvalaki C, Papadakis S, Vardavas C, Pipe A, and C Lionis. Tobacco treatment TrAining Network in Crete (TiTAN-Crete): protocol for a controlled beforeafter study. Tob. Prev. Cessation 2016;2(June):67 doi: $10.18332 / \mathrm{tpc} / 63823$

18. Delucchi KL, Tajima B, Guydish J. Development of the smoking knowledge, attitudes, and practices (S-KAP) instrument. J Drug Issues. 2009;39(2):347-364. doi: 10.1177/002204260903900207

19. Ampt AJ, Amoroso C, Harris MF, et al. Attitudes, norms and controls influencing lifestyle risk factor management in general practice. BMC Family Practice 2009;10:59. doi: 10.1186/1471-2296-10-59

20. Benson F, Nierkens V, Stronks K, Willemsen M. Effects of different types of smoking cessation behavioral therapy in disadvantaged areas in the Netherlands: an observational study. Tob. Prev. Cessation 2016;2:14 doi:10.18332/tpc/61616

21. Martínez C, Castellano Y, Andrés A, Fu M, Antón L, Ballbè M, Fernández P, Cabrera S, Riccobene A, Gavilan E, Feliu A, Baena A, Margalef M, Fernández
E. Factors associated with implementation of the 5A's smoking cessation model. Tob Induc Dis. 2017:15:41. doi: 10.1186/s12971-017-0146-7.

22. Qaseem A, Wilt T, Weinberger SE, et al. Diagnosis and Management of Stable Chronic Obstructive Pulmonary Disease: A Clinical Practice Guideline Update from the American College of Physicians, American College of Chest Physicians, American Thoracic Society, and European Respiratory Society. Annals of Internal Medicine 2011;155(3):179-191. doi: 10.7326/0003-4819-155-3-201108020-00008

23. American Diabetes Association. Standards of medical care in diabetes-2017. Diabetes Care 2017; 40(1): S132-S135. doi: 10.2337/dc17-in01

24. Rydén L, Grant PJ, Anker SD, Berne C, Cosentino F, Danchin N, and M Marre. ESC guidelines on diabetes, pre-diabetes, and cardiovascular diseases developed in collaboration with the EASD-summary. Diabetes \& Vascular Disease Research. 2014:11(3); 133-73. doi: 10.1177/1479164114525548

25. Piepoli MF, Hoes AW, Agewall S. Albus C, Brotons C, Catapano AL, and Graham, I. 2016 European Guidelines on cardiovascular disease prevention in clinical practice: The Sixth Joint Task Force of the European Society of Cardiology and Other Societies on Cardiovascular Disease Prevention in Clinical Practice (constituted by representatives of 10 societies and by invited experts) Developed with the special contribution of the European Association for Cardiovascular Prevention \& Rehabilitation (EACPR). European Heart Journal. 2016;37(29): 23152381. doi: 10.1093/eurheartj/ehw106

26. Mostofian F, Ruban C, Simunovic N, Bhandari M.Changing physician behavior: what works? Am J Manag Care. 2015;21(1):75-84.

27. Carson KV, Verbiest ME, Crone MR, et al. Training health professionals in smoking cessation. Cochrane Database Syst Rev. 2012. CD000214. doi: 10.1002/14651858.CD000214.pub2

28. Papadakis S, McDonald P, Reid RD, Pipe A, Mullen K, and K Skulsky. Strategies to improve the delivery of smoking cessation treatments in primary care: A systematic review and meta-analysis. Prev Med. 2010;51(3-4):199-213. doi: 10.1016/j.ypmed.2010.06.007

29. Purkabiri K, Steppacher V, Bernardy K, Karl N, Vedder V, Borgmann M, Rogausch A, Stammberger U, Bals R, Raupach T, Koellner V, Hamacher J. Outcome of a four-hour smoking cessation counselling workshop for medical students. Tob Induc Dis. 2016;14:37. doi: 10.1186/s12971-016-0103-x

30. Pascal OI, Trofor A, Lotrean M, Filipeanu D, Trofor L. Depression, anxiety and panic disorders in chronic obstructive pulmonary disease patients: correlations with tobacco use, disease severity and quality of life. Tob Induc Dis. 2017;15:23. doi: 10.1186/s12971-017-0128-9

31. Papadakis S, Gharib M, Hambleton J, Reid RD, Assi 
R, and AL Pipe. Delivering evidence-based smoking cessation treatment: The experience of Ontario Family Health Teams. Canadian Family Physician 2014;60:e36271. PMCID: PMC4096282

\section{ACKNOWLEDGEMENTS}

We would like to acknowledge Dr. Larisa Andreea Moldovan, Dr. Cristina Vicol, Dr. Ioana Buculei-Porosnicu, and Vergina Vyzikidou for their contribution to the pilot study implementation. We would also like to thank Dr. Mihaela Ivan and Dr. Ramona Miron for their support.

\section{CONFLICTS OF INTEREST}

Author Panagiotis K. Behrakis is the Editor in Chief of TPC and that there are no conflicts of interest with this current work. The rest of the authors have completed and submitted an ICMJE form for disclosure of potential conflicts of interest. The authors declare that they have no competing interests, financial or otherwise, related to the current work.

FUNDING

This study was funded from the European Union's 3rd Health Programme 20142020, under grant agreement 664292.

PROVENANCE AND PEER REVIEW

Not commissioned;

externally peer reviewed 